\title{
Diagnostic value of carbohydrate antigens in supernatants and sediments of pleural effusions
}

\author{
DANIELA TERRACCIANO ${ }^{1}$, CLAUDIA MAZZARELLA ${ }^{1}$, MARCELLINO CICALESE $^{2}$, \\ SONIA GALZERANO ${ }^{2}$, GIANFRANCO APOSTOLICO ${ }^{2}$, ANGELINA DI CARLO $^{3}$, \\ ANGELA MARIANO $^{1}$, CIRIACO CECERE $^{2}$ and VINCENZO MACCHIA ${ }^{1}$ \\ ${ }^{1}$ Dipartimento di Biologia e Patologia Cellulare e Molecolare 'L. Califano'; ${ }^{2}$ Dipartimento di Chirurgia Toracica - \\ Facoltà di Medicina e Chirurgia, Università degli Studi di Napoli Federico II, 80131 Napoli; \\ ${ }^{3}$ Dipartimento di Medicina Sperimentale, Università di Roma 'La Sapienza', 00161 Roma, Italy
}

Received January 14, 2010; Accepted March 19, 2010

DOI: 10.3892/ol_00000082

\begin{abstract}
A panel of tumour markers including carcinoembryonic antigen (CEA), carbohydrate antigen (Ca)15-3, Ca125 and Ca19-9 were measured in the lysate of sediments and in the supernatants of pleural effusions of patients with benign and malignant disease. The tumour markers were also measured in the serum of the same patients. Of these patients, $32 \mathrm{had}$ benign diseases (12 trasudative effusions associated with cirrhosis and 20 with non-malignant exudates: 12 pleuritis and 8 other inflammations) and 103 had malignant effusions (37 breast cancers, 29 lung cancers, 10 ovary cancers, 6 kidney cancers, 11 mesotheliomas and 10 lymphomas). We showed the highest level of CEA in pleural effusions of lung cancer followed by that in pleural effusions of breast cancer; whereas Ca15-3 was very high in the pleural effusions of breast and lung cancer. Concerning the lysate of sediment, CEA was high in the pleural effusions of patients with lung cancer and Ca15-3 in those of patients with breast cancer. The other markers are much less useful. For the remaining tumours, none of the markers tested appear to aid in the diagnosis of disease. In conclusion, our data suggest that the combined determination of tumour markers on supernatants and sediments of pleural effusion may provide additional information on the nature of pleural effusion, especially for cases with negative cytology.
\end{abstract}

\section{Introduction}

Pleural effusions are classically divided into trasudate and exudate. Trasudate is a liquid that has accumulated as a

Correspondence to: Professor Vincenzo Macchia, Dipartimento di Biologia e Patologia Cellulare e Molecolare 'L. Califano', Università degli Studi di Napoli Federico II, Via Sergio Pansini 5, 80131 Napoli, Italy

E-mail: macchia@unina.it

Key words: pleural effusions, carcinoembryonic antigen, carbohydrate antigen 15-3, carbohydrate antigen 125 result of a systemic illness, such as heart failure or cirrhosis, whereas exudate is generally associated with a localized disorder, involving the pleural surfaces, such as inflammation, a malignant process or an infection. This distinction was based on classic Light's criteria (1). Since Light criteria permit the classification of some pleural fluid, although not always accurately (2-5), fluid to serum protein ratio was suggested (5). The main importance of this trasudate/exudate distinction is in determining the need for subsequent diagnostic tests. If the effusion is trasudate, no other pleural diagnostic action is required, but patients may require other general diagnostic and, of course, therapeutic interventions. If the effusion is exudate, further tests are required to determine its cause.

Malignancy is one of the main causes of pleural effusions and more than $90 \%$ of malignant pleural effusions are due to metastatic disease (6). The most frequent neoplasias that metastasize to the pleura are lung and breast carcinomas and lymphomas, albeit less frequently, as well as digestive and ovarian carcinomas (7). The differential diagnosis of the various malignancies is a clinical and laboratory challenge. Diagnosis is normally carried out by invasive techniques, such as thoracoscopy, which show sensitivity but are not costeffective and induce physical and mental stress in the patient (8). The role of biochemical parameters or tumour markers were previously studied in order to increase the diagnostic capacity of pleural effusion analysis (9-13). The detection of these parameters or markers in bodily fluids is the result of a dynamic balance between the number of cells that synthesize the tumour marker, its capacity for synthesis and the amount eliminated by the organism relative to the nature, size and metabolic mechanism of the marker. However, the exact role of tumour marker assays in differentiating malignant from benign pleural effusion has yet to be elucidated.

Another non-invasive method carried out for the diagnosis of malignant effusion is the cytological examination, which, however, has a sensitivity between 40 and $80 \%$ (14). This discrepancy is caused by various factors, including the quality of the preparations, and the presence of cellular materials of normal tissue and tumour cells, which are very few. Notably, malignant cells will not always appear in the effusions of cancer patients. This is due to the fact that malignant 
Table I. Demographic data and pleural fluids etiology of patients with malignant and non-malignant effusions.

\begin{tabular}{lcccc}
\hline Cause & $\mathrm{n}$ & Gender $(\mathrm{m} / \mathrm{f})$ & Age range (years) & Mean age (years) \\
\hline Malignant & 103 & $41 / 62$ & $22-84$ & 61 \\
Breast cancer & 37 & $0 / 37$ & $32-84$ & 56 \\
Lung cancer & 29 & $23 / 6$ & $43-78$ & 64 \\
Ovarian cancer & 10 & $0 / 10$ & $32-79$ & 60 \\
Kidney cancer & 6 & $2 / 4$ & $48-76$ & 62 \\
Mesotheliomas & 11 & $8 / 3$ & $57-76$ & 64 \\
Lymphomas & 10 & $6 / 4$ & $22-69$ & 55 \\
Non-malignant & 32 & & $37-80$ & 62 \\
Trasudates & & & & 67 \\
Liver cirrhosis & 12 & $14 / 6$ & $63-69$ & 63 \\
Exudates & 20 & $3 / 4$ & $42-80$ & 65 \\
Pleuritis & 12 & $1 / 1$ & $43-69$ & 61 \\
Tuberculosis & 4 & $1 / 0$ & $37-75$ & 64 \\
Pancreatitis & 2 & $1 / 0$ & $60-69$ & 64 \\
Benign asbestos & 1 & 64 & 66 \\
Pleural amyloidosis & 1 & & 66 & \\
\hline
\end{tabular}

disease produces pleural effusion through a series of different mechanisms: lymphatic and capillary destruction, resulting in a reduced absorbency of fluids and proteins; chemical mediators increasing capillary permeability; or atelectasias or erosion of the blood vessels producing malignant disorders (15). In order to improve the diagnostic sensitivity of cytological examination, new approaches were proposed, including the individual or combined cellular neoplastic markers by immunocytochemistry methods (15). However, this method requires an antibody panel able to characterize or distinguish carcinoma cells. The present study aimed to determine the optimal panel of tumour markers in the supernatants and sediments of pleural fluids in order to improve the diagnosis of malignant effusions, particularly in cytologically negative effusions.

\section{Materials and methods}

Serum and pleural effusion samples. Between March 2007 to November 2008, 135 pleural effusions were collected from patients with perfectly defined aetiology from the Surgery Department of our Faculty, and examined. Patient evaluation included anamnesis, physical examination, chest X-ray and thoracocentesis with the biochemical, cytological and bacteriological study of pleural fluid. When the result of the cytological examination was negative or in doubt, patients underwent blind pleural biopsies and/or thoracoscopic-guided biopsies. Pleural effusions exhibited definite aetiologies. Fresh pleural fluid was obtained by thoracocentesis, collected in sterile tubes without anticoagulant and rapidly brought to our laboratory with a blood sample of the same patient. Pleural fluids and blood samples were immediately centrifuged. The supernatants were aliquoted and stored at $-80^{\circ} \mathrm{C}$ and the sediments were partially used immediately for cytological study. The remaining sediments were then partially washed in $0.154 \mathrm{M} \mathrm{NaCl}$ on ice and resuspended in lysis buffer $(40 \mathrm{mM}$ Hepes, 20\% glycerol, 2\% Triton, 2\% Aprotinin and $4 \mathrm{mM}$ EDTA) and stored at $-80^{\circ} \mathrm{C}$, until defrosted and tested for tumour marker content.

Materials. Aprotinin, glycerol, Hepes and Triton were purchased from Sigma Chemical Co. (St. Louis, MO, USA). The BioRad protein assay reagent was from BioRad Laboratories. The LDH monotest and Cholesterol assay reagents were from Diacron Laboratories (Italy). The remaining reagents were available from commercial sources.

Measurement of proteins and tumour markers. Proteins were measured by the Bradford procedure (16). The amount of carcinoembryonic antigen (CEA), carbohydrate antigen (Ca)125, Ca19-9 and Ca15-3 in the blood samples, supernatants of pleural effusions and the lysates of sediments were measured. Tumour markers were determined using the Immulite analyzer, according to the manufacturer's specifications and commercial kits (Diagnostic Products, Los Angeles, CA, USA). Tumour marker contents in the pleural fluids and serum were expressed as $\mathrm{ng} / \mathrm{ml}$ for CEA, whereas Ca15-3, Ca125 and Ca19-9 were expressed as U/ml. CEA was expressed as $\mathrm{ng} / \mathrm{mg}$ of protein in the cellular lysates and Ca15-3, Ca125 and Ca19-9 as U/mg of protein.

Due to the non-normal distribution of the variables, results are expressed as the median and interquartile (IQ) range.

The optimal cut-off of tumour markers in pleural fluids was determined by plotting the true-positive (sensitivity) vs. the false-positive (1-specificity) results in receiver operating characteristic (ROC) curves. An optimal cut-off point was defined as a point on a ROC curve nearest to the point where 
Table II. Biochemical characteristics of pleural fluids (mean \pm SD).

\begin{tabular}{lcccccc}
\hline $\begin{array}{l}\text { Type of pleural } \\
\text { effusion }\end{array}$ & Pathology & $\begin{array}{c}\text { Cholesterol } \\
(\mathrm{mmol} / \mathrm{l})\end{array}$ & $\begin{array}{c}\text { Protein } \\
(\mathrm{g} / \mathrm{l})\end{array}$ & $\begin{array}{c}\text { LDH } \\
(\mathrm{U} / \mathrm{l})\end{array}$ & $\begin{array}{c}\text { Protein pleural } \\
\text { effusion }\end{array}$ & $\begin{array}{c}\text { LDH pleural } \\
\text { effusion }\end{array}$ \\
\cline { 3 - 6 } & & & & Protein serum & LDH serum \\
\hline Trasudate & Cirrhosis & $0.37 \pm 0.21$ & $22.6 \pm 7.2$ & $153 \pm 52$ & $0.37 \pm 0.17$ & $0.65 \pm 0.14$ \\
Benign exudate & Inflammation & $0.82 \pm 0.23$ & $31 \pm 7.2$ & $316 \pm 120$ & $0.57 \pm 0.12$ & $1.40 \pm 0.40$ \\
Malignant exudate & Breast cancer & $1.22 \pm 0.80$ & $43 \pm 15$ & $398 \pm 252$ & $0.69 \pm 0.23$ & $1.06 \pm 0.56$ \\
& Lung cancer & $1.33 \pm 0.87$ & $37 \pm 12$ & $450 \pm 266$ & $0.71 \pm 0.45$ & $0.97 \pm 18.0$ \\
& Ovarian cancer & $1.11 \pm 0.72$ & $43 \pm 12$ & $405 \pm 227$ & $0.74 \pm 31.0$ & $1.06 \pm 22.0$ \\
& Kidney cancer & $1.54 \pm 0.57$ & $46 \pm 17$ & $240 \pm 50$ & $0.6 \pm 0.17$ & $0.81 \pm 0.05$ \\
& Mesotheliomas & $1.15 \pm 76.0$ & $42 \pm 18$ & $272 \pm 190$ & $0.9 \pm 70.0$ & $0.97 \pm 29.0$ \\
& Lymphomas & $0.96 \pm 56.0$ & $37 \pm 10$ & $361 \pm 238$ & $0.61 \pm 14.0$ & $0.91 \pm 29.0$ \\
\hline
\end{tabular}

sensitivity and specificity were $1 . \mathrm{P} \leq 0.05$ was considered to indicate statistical significance. Statistical analyses were performed using the MedCalc software v. 11 statistical program.

\section{Results}

During the 20-month period, a total of 135 patients with pleural effusions were evaluated. Etiological diagnosis and demographic data of patients are shown in Table I. Table II shows the studied biochemical characteristics of the pleural fluids. Using clinical criteria, pleural fluid protein, cholesterol and LDH content were measured, and the pleural fluids were classified into exudates and trasudates. Furthermore, fluid to serum total protein ratio and fluid to serum $\mathrm{LDH}$ ratio analyses were performed. For each parameter, a cut-off for distinguishing exudates from trasudates was previously suggested (5). These cut-off values were also used in this study (for fluid cholesterol $0.8 \mathrm{mmol} / 1$, for fluid protein $28 \mathrm{~g} / 1$, for fluid LDH $380 \mathrm{U} / 1$, for fluid to serum total protein ratio 0.4 and for fluid $\mathrm{LDH}$ to serum $\mathrm{LDH}$ ratio 0.9 ). The five parameters allowed for the differentiation of exudates from trasudates. Furthermore, the mean values $( \pm$ SD) of fluid cholesterol, fluid protein and the fluid protein/serum protein ratio were higher in the malignant than in the benign fluids, whereas the fluid $\mathrm{LDH} /$ serum $\mathrm{LDH}$ ratio was higher in the benign than in the malignant fluids. The four tumour markers were measured in the serum and pleural fluid of patients with benign and malignant disease. Results were expressed as the median and IQ range (Table III).

CEA levels in the serum of patients with breast and lung cancers were 8- and 20-fold higher, respectively, than those in the serum of patients with benign disease. In patients with ovarian cancers and lymphomas, CEA serum levels were slightly higher than those in patients with benign disease, but no difference between CEA serum levels in subjects with kidney cancers, mesotheliomas and benign disease was found. In patients with breast, lung and ovarian cancers, the CEA pleural effusion levels were much higher than those in the pleural fluids of patients with benign disease and slightly higher in the pleural effusions of patients with ovarian cancers and lymphomas. Values similar in serum and pleural effusions of CEA levels in subjects with kidney cancers, mesotheliomas and benign disease were found.

In patients with breast, lung, ovarian cancers and lymphomas, serum and pleural effusion Ca15-3 levels were much higher than those in the pleural fluids of patients with benign disease, but not so high in patients with kidney cancer and mesotheliomas. Concerning Ca125, the serum levels were similar in patients with benign and malignant disease. However, 6-fold higher levels in the pleural effusions of patients with breast and lung cancer related to those of patients with benign disease were observed. Ca125 values in the pleural effusions of patients with ovarian cancer and lymphomas were slightly higher than those in the pleural fluids of patients with benign disease. On the other hand, Ca125 values were similar to those of the patients with benign disease in the pleural fluids of patients with mesotheliomas and kidney cancer. No differences in the serum values of Ca19-9 in patients with benign disease nor in patients with breast, lung, kidney cancers, mesotheliomas and lymphomas were observed. However, in the serum of patients with ovarian cancers, the Ca19-9 values were 10-fold higher than those found in the pleural fluids of patients with benign disease. However, these values were not noted in the pleural effusions of patients with kidney cancer, lymphomas and mesotheliomas. On the other hand, Ca19-9 values were between 6- and 40-fold higher than those of the patients with benign disease in the remaining pleural effusions of patients with malignant disease (breast, lung and ovarian cancers). The percentage of positivity for CEA, Ca15-3 and Ca125 in breast, lung and ovarian cancer pleural fluids was also calculated, whereas any data pertaining to Ca19-9 were no longer considered. The term 'positive' was considered for values equal or higher to the cut-off values. Since normal pleural fluids were not available, the cut-off values for the three markers were calculated using tumour marker values of pleural fluids of patients with benign exudates. The optimal cut-off points for pleural fluid were obtained by ROC-curve 


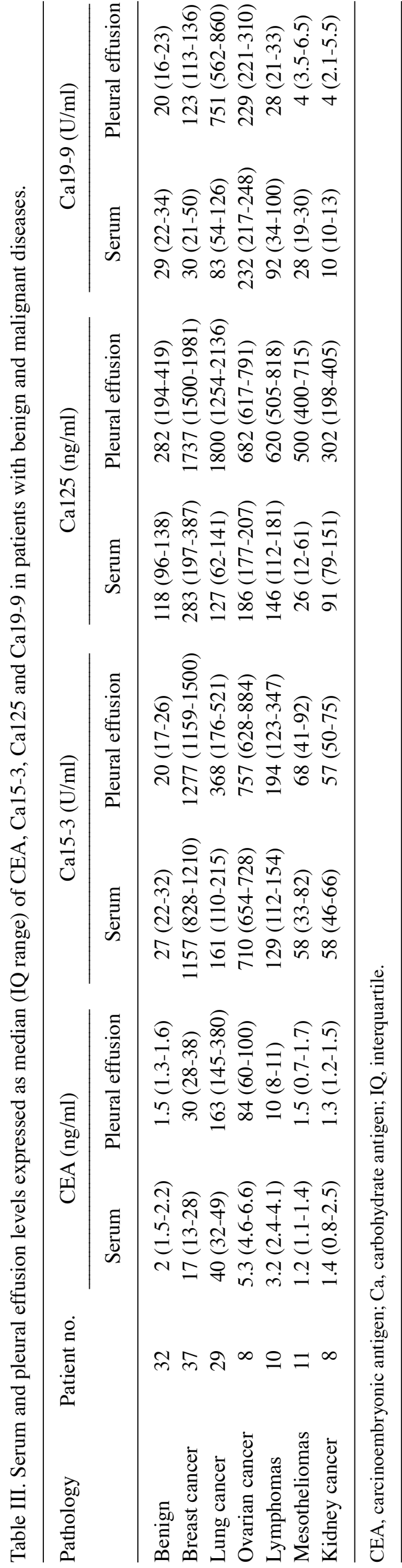

Table IV. Optimal cut-off point of tumour markers in pleural effusion.

\begin{tabular}{lrcc}
\hline & Cut-off point & Sensitivity (\%) & Specificity (\%) \\
\hline CEA & $2.1 \mathrm{ng} / \mathrm{ml}$ & 82 & 100 \\
Ca15-3 & $41 \mathrm{U} / \mathrm{ml}$ & 96 & 100 \\
Ca125 & $459 \mathrm{U} / \mathrm{ml}$ & 82 & 96 \\
\hline
\end{tabular}

CEA, carcinoembryonic antigen; $\mathrm{Ca}$, carbohydrate antigen.

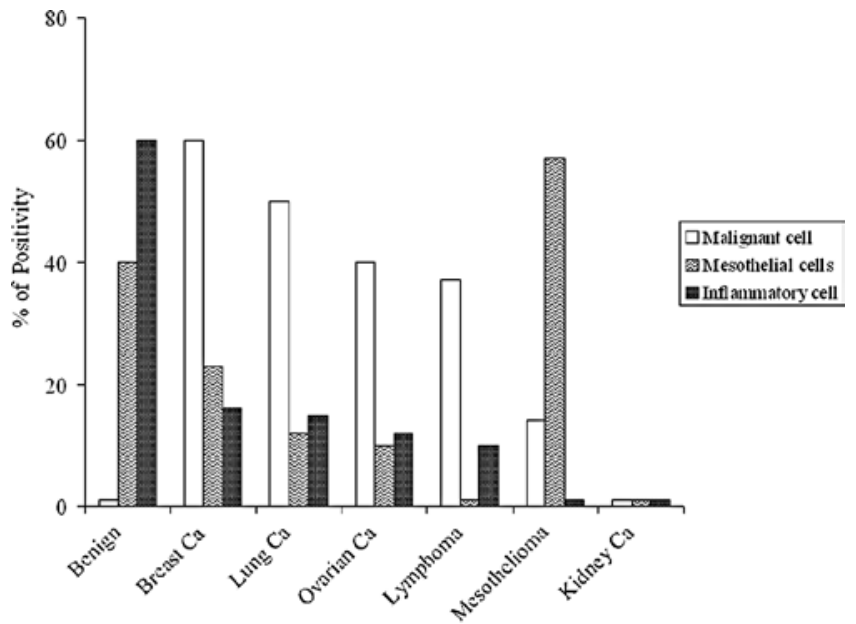

Figure 1. Positivity (\%) of malignant, mesothelial and inflammatory cells in the pleural effusion sediments.

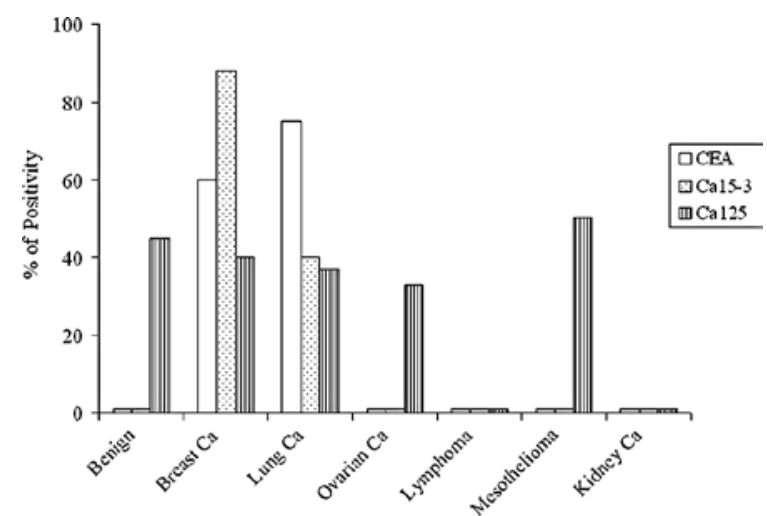

Figure 2. Positivity (\%) of CEA, Ca15-3 and Ca125 in the crude extracts of pleural effusion sediments.

analyses (Table IV). The optimal cut-off values for pleural fluid were CEA $2.1 \mathrm{ng} / \mathrm{ml}$, Ca15-3 $41 \mathrm{U} / \mathrm{ml}$ and Ca125 $459 \mathrm{U} /$ $\mathrm{ml}$. Using these cut-off values, CEA and Ca15-3 were found to be positive in all samples (37/37) of the pleural effusions of breast cancer patients, and Ca125 was positive in 34/37 (90\%) of the total samples of pleural effusions. In lung cancers, CEA and Ca15-3 were positive in $23(80 \%)$ and $20(70 \%)$ of the total samples of pleural effusions, respectively, and Ca125 was positive in $20(70 \%)$ of the total samples of pleural effusions. All samples of pleural fluids analyzed were positive for CEA and Ca15-3 in ovarian cancers. 
Table V. Presence of malignant cells and expression of tumour markers in packed materials lysates of pleural fluids of single patients with breast cancer.

\begin{tabular}{|c|c|c|c|c|}
\hline \multirow[t]{2}{*}{ Patient no. } & \multirow[t]{2}{*}{$\begin{array}{l}\text { Malignant } \\
\text { cells }\end{array}$} & \multicolumn{3}{|c|}{$\begin{array}{l}\text { Expression of } \\
\text { tumour markers }\end{array}$} \\
\hline & & CEA & Ca15-3 & Ca125 \\
\hline 1 & - & + & + & - \\
\hline 2 & + & - & + & - \\
\hline 3 & + & + & + & + \\
\hline 4 & + & + & + & - \\
\hline 5 & - & - & + & - \\
\hline 6 & + & + & + & + \\
\hline 7 & - & - & + & - \\
\hline 8 & - & - & - & + \\
\hline 9 & + & + & + & + \\
\hline 10 & - & - & + & - \\
\hline 11 & - & - & + & - \\
\hline 12 & - & + & + & - \\
\hline 13 & - & - & - & + \\
\hline 14 & + & + & + & - \\
\hline 15 & + & - & + & + \\
\hline 16 & + & + & + & - \\
\hline 17 & - & + & - & + \\
\hline 18 & + & + & + & - \\
\hline 19 & - & - & + & - \\
\hline 20 & - & - & - & + \\
\hline 21 & + & + & + & - \\
\hline 22 & + & + & + & - \\
\hline 23 & + & + & + & - \\
\hline 24 & + & + & + & + \\
\hline 25 & + & + & + & - \\
\hline 26 & + & + & + & + \\
\hline 27 & - & - & + & - \\
\hline 28 & + & + & + & - \\
\hline 29 & - & - & - & + \\
\hline 30 & + & + & + & - \\
\hline 31 & - & - & + & - \\
\hline 32 & + & + & + & + \\
\hline 33 & + & - & + & + \\
\hline 34 & + & + & + & - \\
\hline 35 & + & + & + & + \\
\hline 36 & + & + & + & - \\
\hline 37 & - & - & + & + \\
\hline
\end{tabular}

CEA, carcinoembryonic antigen; Ca, carbohydrate antigen.

The pleural effusions were immediately centrifuged and a cytologic study was performed; Fig. 1 shows the results. The highest percentage of malignant cells was found in the pleural effusions of patients with breast cancers $(60 \%)$, followed by those with lung cancers (50\%), ovarian cancers (40\%) and lymphomas (37\%). The highest percentage of positivity for the inflammatory cells was found in the samples of benign pleural
Table VI. Presence of malignant cells and expression of tumour markers in packed materials lysates of pleural fluids of single patients with lung cancer.

\begin{tabular}{|c|c|c|c|c|}
\hline \multirow[t]{2}{*}{ Patient no. } & \multirow[t]{2}{*}{$\begin{array}{l}\text { Malignant } \\
\text { cells }\end{array}$} & \multicolumn{3}{|c|}{$\begin{array}{l}\text { Expression of } \\
\text { tumour markers }\end{array}$} \\
\hline & & CEA & Ca15-3 & Ca125 \\
\hline 1 & - & + & - & + \\
\hline 2 & - & + & - & + \\
\hline 3 & + & + & + & - \\
\hline 4 & + & + & - & - \\
\hline 5 & + & + & + & + \\
\hline 6 & + & + & - & - \\
\hline 7 & - & - & + & - \\
\hline 8 & - & + & + & - \\
\hline 9 & - & + & - & + \\
\hline 10 & - & + & - & - \\
\hline 11 & + & + & + & - \\
\hline 12 & + & + & + & + \\
\hline 13 & + & + & - & - \\
\hline 14 & + & + & + & - \\
\hline 15 & - & - & - & - \\
\hline 16 & - & - & - & + \\
\hline 17 & - & - & - & + \\
\hline 18 & + & + & + & - \\
\hline 19 & - & + & + & - \\
\hline 20 & + & + & - & - \\
\hline 21 & - & - & - & + \\
\hline 22 & + & + & - & + \\
\hline 23 & + & + & + & - \\
\hline 24 & - & + & + & - \\
\hline 25 & + & + & + & - \\
\hline 26 & + & + & - & - \\
\hline 27 & - & - & - & + \\
\hline 28 & - & - & - & + \\
\hline 29 & + & + & - & - \\
\hline
\end{tabular}

effusions $(60 \%)$. Low percentage of positivity or negativity was observed for the remaining samples. Mesotheliomas showed the highest percentage for mesothelial cells (57\%). However, $40 \%$ of samples from benign pathologies were also positive for mesothelial cells. Low percentages of positivity or negativity were found for the remaining samples. CEA, Ca15-3 and Ca125 were measured on all the lysates obtained; Fig. 2 shows the results. In these materials, a marker was considered positive when it was detected (values higher than the analytical sensitivity of the method). Ca125 was positive in $50 \%$ of samples from mesotheliomas, followed by benign pathologies (45\%), breast (40\%), lung (37\%) and ovarian cancers $(33 \%)$, negative kidney cancer and lymphomas. CEA and $\mathrm{Ca} 15-3$ were undetectable in benign samples, mesotheliomas, lymphomas and ovarian and kidney cancers. The 
highest percentage of Ca15-3 positivity was observed in breast cancers $(88 \%)$, followed by lung cancers $(40 \%)$, whereas CEA was very high in lung cancers $(75 \%)$, followed by breast cancers $(60 \%)$. For breast and lung cancers, the percentage of positivity of the three markers was compared to the presence of malignant cells in individual patients; Tables $\mathrm{V}$ and VI show the results. The means \pm SE of the three markers were calculated using only the positive samples and the data were expressed as ng or $\mathrm{U} / \mathrm{mg}$ of proteins. In breast cancer samples, the three markers values were: CEA, $131 \pm 40 \mathrm{ng} / \mathrm{mg}$; $\mathrm{Ca} 15-3,305 \pm 77 \mathrm{U} / \mathrm{mg}$ and $\mathrm{Ca} 125,184 \pm 77 \mathrm{U} / \mathrm{mg}$ proteins. In lung cancers samples, the following data were found: CEA, $348 \pm 71 \mathrm{ng} / \mathrm{mg}$; Ca15-3, 86 $\pm 21 \mathrm{U} / \mathrm{mg}$ and Ca125, 204 $\pm 96 \mathrm{U} / \mathrm{mg}$ proteins. On the other hand, the three markers in lymphomas and kidney cancer samples were undetectable, whereas Ca125 had high values in ovarian cancers $(339 \pm 124 \mathrm{U} / \mathrm{mg}$ proteins) and in mesotheliomas (219 $\pm 61 \mathrm{U} / \mathrm{mg}$ proteins).

\section{Discussion}

Pleural effusions are common complications of a wide variety of diseases. To elucidate their precise etiologies and to differentiate malignant from non-malignant effusions, the laboratory plays an important role.

Cytological analysis (17) remains the main diagnostic approach. Diagnosis of malignancy is well established when neoplastic cells are found in pleural fluids. A point to be considered is whether the cells are local or metastatic cancer cells. If the cells are metastatic then the organ they originate from needs to be located. Of note, however, is that in $40-80 \%$ of the malignant effusions the cytological analysis depends on the investigator's experience (18). Therefore, in order to obtain more information from laboratory analyses, certain parameters were measured in pleural fluid and serum in order to determine trasudates and exudates. Furthermore, we measured a large panel of tumour markers on lysate extracts of the sediments of the pleural effusions, in the serum and in the supernatant of pleural effusions, obtained from the same patients. Our study showed that it is easy to differentiate exudates from trasudates by measuring fluid choleseterol, protein and LDH and fluid to serum total protein ratio, as well as fluid LDH to serum LDH ratio. Additionally, we showed that fluid cholesterol, fluid protein and the fluid protein/serum protein ratio were higher in the malignant than in benign fluids, whereas fluid LDH/fluid serum ratio was higher in the benign than in malignant fluids. In agreement with other authors (19), pleural fluid CEA levels in patients with lung cancers were significantly higher than those in patients with benign disease, with $100 \%$ of positivity rate at a cut-off level of $2.1 \mathrm{ng} / \mathrm{ml}$. In addition, $75 \%$ of the cytosolic materials of pleural effusion sediments were positive for CEA, whereas the cytological analysis detected neoplastic cells in only $50 \%$ of the samples. These results strongly suggest that the combined use of CEA determination on pleural effusions and on cytosolic materials of the pleural effusion sediments is more useful than the cytological analysis. Our data showed the remaining markers in pleural effusions and sediments to be less useful for this malignancy. Pleural fluid Ca15-3 levels in patients with breast cancers were significantly higher than those in patients with benign disease, with $100 \%$ of positivity rate at a cut-off level of $41 \mathrm{U} / \mathrm{ml}$. In addition, $88 \%$ of the cytosolic materials of pleural effusion sediments were positive for Ca15-3, whereas the cytological analysis detected neoplastic cells in only $60 \%$ of the samples. Our data indicated that the other markers are less useful for breast cancer in pleural effusions and on cytological materials of pleural effusion sediments. On the other hand, using cytological analysis and tumour marker measurements, the percentage of positivity was not higher than $50 \%$ of the samples for ovarian cancers. Regarding the remaining malignant diseases (kidney cancer, lymphomas and mesotheliomas), our results did not indicate any analytical test to be useful for the diagnosis of these diseases.

Furthermore, in agreement with other authors $(12,20)$, Ca125 is not recommended as a useful diagnostic tool in malignant pleural effusion since immunohistochemical studies have shown that Ca125 is released from the pleura as well as from the peritoneum. In the present study, when the percentage of positivity for Ca125 was considered in the cytosolic materials of pleural effusion sediments, no significant difference was observed between benign and malignant diseases. Finally, Ca19-9, as well as Ca125, do not aid in the cytological diagnosis of pleural effusions. Taking together these observations, we suggest that: i) the tumour markers CEA and Ca15-3 measured in pleural fluids may provide clinicians with additional information on the nature of pleural fluids; ii) Ca15-3 and CEA appear to be good indicators for breast and lung cancer, respectively, and finally iii) the use of cytosols of pleural effusion sediments of tumour markers is very useful, especially in cytologically negative cases.

\section{References}

1. Light RW, Macgregor MI, Luchsinger PC and Ball WC Jr: Pleural effusions: the diagnostic separation of transudates and exudates. Ann Intern Med 77: 507-513, 1972.

2. Gazquez I, Porcel JM, Vives M, Vicente de Vera MC, Rubio M and Rivas MC: Comparative analysis of Light's criteria and other biochemical parameters for distinguishing transudates from exudates. Respir Med 92: 762-765, 1998.

3. Paramothayan NS and Barron J: New criteria for the differentiation between transudates and exudates. J Clin Pathol 55: 69-71, 2002.

4. Heffner JE, Sahn SA and Brown LK: Multilevel likelihood ratios for identifying exudative pleural effusions $\left({ }^{*}\right)$. Chest 121 : 1916-1920, 2002

5. Terracciano D, Di Carlo A, Papa P, Cicalese M, Maietta P, Cecere C, Mariano A and Macchia V: New approaches in the diagnostic procedure of malignant pleural effusions. Oncol Rep 12: 79-83, 2004.

6. Fenton KN and Richardson JD: Diagnosis and management of malignant pleural effusions. Am J Surg 170: 69-74, 1995.

7. Marel M, Stastny B, Melinova L, Svandova E and Light RW: Diagnosis of pleural effusions. Experience with clinical studies, 1986 to 1990. Chest 107: 1598-1603, 1995.

8. Menzies R and Charbonneau M: Thoracoscopy for the diagnosis of pleural disease. Ann Intern Med 114: 271-276, 1991.

9. San Jose ME, Alvarez D, Valdes L, Sarandeses A, Valle JM and Penela P: Utility of tumour markers in the diagnosis of neoplastic pleural effusion. Clin Chim Acta 265: 193-205, 1997.

10. Paganuzzi M, Onetto M, Marroni P, Filiberti R, Tassara E, Parodi S and Felletti R: Diagnostic value of CYFRA 21-1 tumor marker and CEA in pleural effusion due to mesothelioma. Chest 119: 1138-1142, 2001.

11. Galbis Caravajal JM, Benlloch Carrion S, Sanchez Paya J, Mafe Madueno JJ, Baschwitz Gomez B and Rodriguez Paniagua JM: Prognostic value of the carcinoembryonic antigen found in pleural lavage fluid from patients with lung carcinoma. Arch Bronconeumol 41: 185-188, 2005. 
12. Ghayumi SM, Mehrabi S, Doroudchi M and Ghaderi A: Diagnostic value of tumor markers for differentiating malignant and benign pleural effusions of Iranian patients. Pathol Oncol Res 11: 236-241, 2005.

13. Antonangelo L, Vargas FS, Seiscento M, Bombarda S, Teixera L and Sales RK: Clinical and laboratory parameters in the differential diagnosis of pleural effusion secondary to tuberculosis or cancer. Clinics 62: 585-590, 2007.

14. Fetsch PA and Abati A: Immunocytochemistry in effusion cytology: a contemporary review. Cancer 93: 293-308, 2001.

15. Kjeldsberg C and Knight J: Pleural and Pericardial Fluids Body Fluids. 3rd edition. ASCP, Chicago, pp159-222, 1993.

16. Bradford MM: A rapid and sensitive method for the quantitation of microgram quantities of protein utilizing the principle of protein-dye binding. Anal Biochem 72: 248-254, 1976.
17. Bedrossian CW: Diagnostic problems in serous effusions. Diagn Cytopathol 19: 131-137, 1998

18. Motherby H, Nadjari B, Friegel P, Kohaus J, Ramp U and Bocking A: Diagnostic accuracy of effusion cytology. Diagn Cytopathol 20: 350-357, 1999.

19. Kuralay F, Tokgoz Z and Comlekci A: Diagnostic usefulness of tumour marker levels in pleural effusions of malignant and benign origin. Clin Chim Acta 300: 43-55, 2000.

20. Shitrit D, Zingerman B, Shitrit AB, Shlomi D and Kramer MR: Diagnostic value of CYFRA 21-1, CEA, CA 19-9, CA 15-3 and CA 125 assays in pleural effusions: analysis of 116 cases and review of the literature. Oncologist 10: 501-507, 2005. 